\title{
Religion und gesellschaftlicher Wandel im Finnland der 60er und Anfang der 70er Jahre des 20. Jahrhunderts
}

\author{
Von VOITTO HUOTARI
}

Die Umwandlung der Gesellschaftsstruktur ist in den 60er Jahren und Anfang der 70er Jahre in Finnland heftiger gewesen als in vielen anderen westeuropäischen Ländern. Hunderttausende von Finnen der jungen und mittleren Jahrgänge sind aus ländlichen Gegenden in die Städte gezogen. Die Industriezentren sind sehr schnell gewachsen. Eine große Anzahl von Gesellschaftsgliedern ist vielen gleichzeitigen Lebensveränderungen ausgesetzt gewesen: Ländliches Gemeinwesen hat sich in städtisches verwandelt, Wohnformen haben sich verändert, desgleichen Arbeitsplatz und Beruf. Man mußte sich an ein neuartiges Netzwerk und Wertgefüge sozialer Beziehungen anpassen.

Im Folgenden werden einige Charakteristika der Stellung von Religion inmitten des finnischen Gesellschaftswandels der 60er und 70er Jahre umrissen. Die Analyse könnte auf der Ebene der Gesamtgesellschaft oder der religiösen Organisationen und Bewegungen oder auf der Ebene der Individuen vorgenommen werden. Wir untersuchen im Folgenden einige Charakteristika auf den beiden zuerst genannten Ebenen, im besonderen aber, wie sich der soziale Wandel in den religiösen Bewegungen, die innerhalb der lutherischen Volkskirche tätig sind, widergespiegelt hat.

Wir fragen also im Folgenden, wie der soziale Wandel im Finnland der 60er und 70er Jahre auf die religiösen Bewegungen gewirkt hat, was für Dynamik und Institutionalisierung jener in ihnen verursacht hat und ob generell religiös darauf reagiert worden ist.

\section{Der soziale Wandel unter dem Gesichtspunkt das religiösen Systems}

Im Zusammenhang mit sozialen Veränderungen ist untersucht worden, wie Religion die Veränderung vorantreibt oder hindert. Die zweite Unter- 
suchungsperspektive ist, wie sich Religion selbst an die Veränderung anpaßt. In Finnland wie auch andererorts in westlichen Ländern wird Religion in ihren traditionellen Formen nicht für ein Wegbereiter gesellschaftlicher Veränderung gehalten, wenigstens nicht in Hinsicht auf zentrale Belange der Gesellschaft ${ }^{1}$. Im allgemeinen wird nämlich der Anteil von Religion als Veränderungen hemmende oder wenigstens verlangsamende Kraft für viel wichtiger gehalten. Da Religion Gesellschaftsintegration wirkt, kann sie nicht gleichzeitig auf Veränderung des herrschenden Systems abzielende Bestrebungen unterstützen². Die Entwicklung auf anderen Bereichen ist vielfach schneller als auf dem Gebiet religiöser Betätigung. Hierin eben wird denn auch häufig gerade die gesellschaftliche Funktionalität von Religion enthalten gesehen. Da Religion sich langsam und später verändert, wirkt sie Kontinuität und hilft den Individuen beim Anpassen an schnelle Veränderungen: Religion lindert den Druck, den Veränderungen mit sich bringen.

Die im religiösen System selbst stattfindenden Veränderungen sind ihrerseits Anpassung an veränderte Umweltbedingungen. So ist es denn aufschlußreich, im besonderen zu untersuchen, wie das religiöse System auf die Umweltveränderung der $60 \mathrm{er}$ und 70er Jahre reagiert hat: Wie hat es sich daran angepaßt und wie hat es andererseits als anpassender Faktor fungiert? Vom Standpunkt religiöser Betätigung her sind im gesellschaftlichen Veränderungsprozeß $\beta$ besonders drei beachtenswerte Züge:

1.1. Die Zunahme von Arbeitsteilung. Die Arbeitsteilung verschiedener Institutionen ist in städtischen Gemeinwesen viel differenzierter als auf dem Lande ${ }^{3}$. In Städten hat denn auch Religion ihren eigenen Stellenwert in erster Linie als Katalysator von Fragen über den Sinn von Leben und Tod bekommen. Die organisatorischen Verbindungen zwischen Religion und anderen sozialen Institutionen haben sich im Verlauf des Veränderungsprozesses abegeschwächt. So ist es auch in Finnland geschehen. In den $60 \mathrm{er}$ und 70er Jahren wurde andauernd öffentlich über das Abbrechen von organisatorischen Verbindungen zwischen Religion und der übrigen Gesellschaft debattiert: über die Stellung von Religion im Schulwesen, über den Anteil der Kirche an sozialer Fürsorge, usw. Die Differenzierung und In-

\footnotetext{
${ }^{1}$ Cf. Luckmann $21 \mathrm{ff}$; Yinger $485 \mathrm{ff}$.

${ }^{2}$ Cf. Glock $170 \mathrm{ff}$.

${ }^{3}$ Cf. Seppänen 163.
} 
stitutionalisierung von Religion und anderen Teilbereichen der Gesellschaft in eigene, abgesonderte Sektoren ist nicht bedeutend für die Stellung von Religion, gesetzt den Fall daß starke Wechselbeziehungen zwischen Religion und anderen Institutionen erhalten bleiben. Die Veränderung hat jedoch auch Abschwächung der Wechselbeziehungen zwischen Religion und anderen Institutionen bedeutet.

1.2. Der Wandel der Struktur von Wechselbeziehungen zwischen Individuen. Wie bekannt, ist der Wandel beschrieben worden als Umwandlung der Gemeinschafts- in Gesellschaftskörperschaften. In dieser Charakterisierung ist der Gedanke davon enthalten, daß sich der Kontakt zwischen Individuen umgestaltet. Vom Standpunkt religiöser Betätigung her ist es wesentlich gewesen, daß die Dorfgemeinschaft ihre Bedeutung eingebüßt hat, wie ja im allgemeinen die auf Territorialität basierenden Gemeinschaften. Zwischenmenschliche Beziehungen werden mehr aufgrund von funktionellen Niveaus als nach Nachbarschaft o. ä. physischer Nähe geschlossen. Religion hat einen eigenen Platz in ländlichen Dorfgemeinschaften gehabt, aber das trifft auf die verschiedenen funktionellen Niveaus städtischer Gemeinwesen in weit geringerem Maße zu.

1.3. Der Wandel von Werten und Normen. Religion hat keine so unmittelbare Beziehung zu politischer oder wirtschaftlicher o. ä. Aktivität der Gesellschaft wie zu Werten und Normen. Die ideologischen, ideellen Veränderungen sind vom Standpunkt der Religion her am aller wesentlichsten gewesen ${ }^{4}$. Dennoch sind sie keine autonomen Geschehnisse, sondern stehen in Zusammenhang mit den Veränderungen anderer sozialer, politischer und wirtschaftlicher Aktivität. Der Wandel von zur Religion gehörigen Werten und Normen wird gewöhnlich Säkularisation genannt. Es herrscht wohl Übereinstimmung darüber, daß Religion in ihrer tradiotionellen Form wenigstens in westlichen Ländern in gewissem Maße ihre Bedeutung verliert. Wenn wir beispielsweise wie Thomas Luckmann das Weltbild von Religion als Sinnhierarchie definieren, können wir sagen, daß den Menschen solch eine stets existent ist und im weiteren, daß in westlichen Ländern Religion lediglich neuartige Formen annimmt ${ }^{5}$. In diesem Zusammenhang lohnt es sich gewiß nicht, weiter auf die Frage nach der Beschaffenheit von Religion einzugehen. Wenn wir ausschließlich ihre

\footnotetext{
${ }^{4}$ Siehe um sozialkulturellen Determinanten religiöser Orientierungen: Singer $170 \mathrm{ff}$.

${ }^{5}$ Cf. Luckmann $33 \mathrm{ff}$.
} 
traditionellen Formen untersuchen, so stellt sich heraus, daß deren Werte und Normen schwächer geworden sind. Gleich nach dem Kriege hat sich in dieser Beziehung ein entscheidender Wandel ereignet, wie auch in den 60 er Jahren. Dieser bewirkte Reaktionen im religiösen System vieler westlicher Länder. So waren denn die Reaktionen in Finnland teils dieselben wie auch anderswo, teils aber erhielten sie ein eigenes Gepräge durch die außerordentliche Heftigkeit des Gesellschaftswandels wie auch durch die besondere Beschaffenheit des finnischen religiösen Feldes.

\section{Institutionalisierte Kirchlichkeit}

Im allgemeinen ist in Finnland wie auch anderweitig auf den sozialen Wandel religiös nicht reagiert worden. Vielmehr hat der Wandel Verminderung der kirchlichen Aktivität bedeutet. In der 60er und 70er Jahren hat sich die Anzahl der Teilnehmer an religiöser Betätigung verringert. Am Anfang der 60er Jahre war die gottesdienstliche Aktivität auf einem seit dem zweiten Weltkrieg unerreichten Höhepunkt: an normalen Sonntagen besuchten ungefähr 130000 Gemeindeglieder den lutherischen Gottesdienst. Das beträgt 3,0\% der Mitglieder aller Gemeinden Finnlands. Seit dem Anfang der 60er Jahre aber ereignete sich ein relativ steiles Absinken der Zahlen. Anfang der 70er Jahre waren dann an normalen Sonntagen nur noch ungefähr 100000 Gottesdienstbesucher zu verzeichnen. Das beträgt 2,4\% aller Gemeindeglieder. Mitte der 70er Jahre ist die Beteiligung wieder etwas angestiegen. Die Aktivität der Teilnahme am Abendmahl hat jedoch die ganze Zeit hindurch recht stark zugenommen. Dies ist wohl dahingehend zu interpretieren, daß sich die Schar der an kirchlicher Aktivität Teilnehmenden verschmälert hat, dagegen aber die Intensität der Beteiligung offensichtlich in gewissem Maße gewachsen ist ${ }^{6}$.

Mit den Lebensphasen des Individuums verknüpfte Kulthandlungen: Taufe, Konfirmation, Trauung und Beerdigung haben dagegen ihre Stellung wohl besser als in vielen anderen Ländern erhalten. Die Kindtaufe wird kaum vernachlässigt. Relativ wenige versäumen den Konfirmandenunterricht. Lediglich auf dem Bereich der kirchlichen Eheschließungen hat sich seit Anfang der 70er Jahre Veränderung bemerkbar gemacht: ein Teil der lutherischen Kirchenangehörigen läßt nämlich die Eheschließung standes-

\footnotetext{
${ }^{6}$ Andere Äußerungen der Religiosität und Kirchlichkeit in Finnland: Koskelainen $110 \mathrm{ff}$; Seppänen $150 \mathrm{ff}$.
} 
amtlich vornehmen. (In Finnland hat nämlich die Kirche das Recht, Eheschließungen zu legalisieren.) Andererseits wird in vielen Fällen christliche Beerdigung auch für jene erbeten, die keine Kirchenmitglieder sind.

Da nichtsdestoweniger das Interesse am zentralsten kirchlichen Tätigkeitsbereich, dem Gottesdienst, nachgelassen hat, kann die allgemeine Entwicklung als eine Art von Polarisierung angesehen werden. Klarer als zuvor hat sich eine Aufspaltung ereignet in an kirchlicher Aktivität Teilnehmende und andernteils Entfremdete, die kein Interesse am kirchlichen Lebenskreis haben. Aber gleichzeitig hat sich auch innerhalb des religiösen Feldes Polarisierung ereignet. Einesteils will man das religiöse System an den Wandel anpassen und sich somit diesem unterwerfen, andernteils wird der Unterwerfung und Anpassung schroffer als zuvor entgegengewirkt.

Eine erste Reaktion auf den gesellschaftlichen Wandel war in den $60 \mathrm{er}$ Jahren die radikale Studentenbewegung ${ }^{7}$. Kirchlicherseits äußerte sich dieser in Form von Forderungen nach gesellschaftlicher Gerechtigkeit, die ausgesprochen religiös motiviert wurden. Eine radikale christliche Studentenbewegung war in Finnland ihren Grundzügen nach ebenso beschaffen wie in anderen westlichen Ländern ${ }^{8}$, von deren adäquaten Bewegungen sie teilweise ideell abhängig war. Erwähnenswerte, soziologisch interessante Ausprägungsformen hat sie offensichtlich nicht gehabt. Die Bewegung bediente sich der für die christliche Aktivität der Studenten konstituierten Organisationen. Ihre Aktivität war auch in Finnland mit der marxistischen Arbeiterbewegung und den darin stattfindenden Veränderungen verbunden. Die Institutionalisierung der radikalen christlichen Studentenbewegung blieb ziemlich schwach. Eine eigentliche Volksbewegung ist nie daraus geworden. Am Anfang der 70er Jahre hat sie sich praktisch gesehen aufgelöst. Die Auflösung geschah in zwei Richtungen: ein Teil ist zur traditionellen Kirchlichkeit zurückgekehrt und der andere zu gesellschaftlicher Aktivität ohne religiöse Motivation übergegangen.

Auch konservative religiöse Reaktionen zeigten sich zunächst in der Welt der Studenten'. So spaltete sich die christliche Studentenbewegung am Anfang der 60er Jahre in zwei Hälften, als eine Organisation namens Studentenmission gegründet wurde. Auch diese Bewegung hat Gemeinsamkeiten und sogar Verbindung mit universaler fundamentalistischer Aktivität

\footnotetext{
7 Cf. Lindqvist 492.

${ }^{8}$ Siehe z. B.: Hollinger $337 \mathrm{ff}$.

${ }^{9}$ Cf. Lindquist 492.
} 
und allgemein mit angelsächsischer Erweckungschristlichkeit. Die Organisierung der Studentenmission bedeutete den Anfang für eine ausgedehntere, auf pietistischer Basis entstandene Erweckungsbewegung und deren Organisierung.

Sowohl religiös radikale als auch konservative Reaktionen waren die ersten Reflexionen von Phänomenen, die der gesellschaftliche Wandel in der mit Kirchlichkeit verbundenen Religiosität verursachte. Bereits darin zeigte sich die oben erwähnte Polarisierung in sich Anpassende hüben und Bewahrende drüben. Anpassende Reaktionen waren am aller extremsten gerade in der Welt der Studenten. Andersartige, auf Anpassung des religiösen Systems an veränderte Verhältnisse abzielende Betätigung hat sich in der Zunahme sozialethischer Aktivität, in der Ausweitung kirchlicher Familienberatung, im Durchbruch der sogen. mitmenschlich zentrierten Seelsorgeperspektive und ähnlichem mehr gezeigt. Dergleichen ist - anders als die radikale Studentenbewegung - kirchlicherseits allgemein akzeptiert worden.

Konservative Reaktionen dagegen erhielten ihren schroffsten Ausdruck anderswo als in der Welt der Studenten. Neben anpassender Tätigkeit spitzten sich im kirchlichen Leben ziemlich ersichtlich Tendenzen zu, der Anpassung zu widerstehen und die Verschiedenartigkeit des religiösen und gesellschaftlichen Wertgefüges zu betonen. Augenscheinlich haben hieran besonders die innerkirchlichen Erweckungsbewegungen teil gehabt.

\section{Volksmission}

Ihrer Wirkung nach die umfangreichste Reaktion im vom Wandel der Gesellschaft bewirkten neuen Zustand stellte die Organisierung einer neuen Erweckungsbewegung dar. Für das finnische religiöse Feld sind stets pietistische, innerhalb der Kirche wirkende Bewegungen spezifisch gewesen, welche dem 17. und 18. Jhrt. entstammen. Diese sind die pietistische Bewegung, die evangelische Bewegung, die laestadianische Bewegung und die Beterbewegung ${ }^{10}$. Die an deren Seite getretene neue, sogen. fünfte Erweckungsbewegung, ist ihrer Natur nach gleichfalls pietistisch. In deren Institutionalisierung spiegelt sich klar die ideelle und sozialökonomische Entwicklung der 60er und 70er Jahre wider.

${ }^{10}$ Cf. Sentzke $101 \mathrm{ff}$; Seppänen 145; Sihvo $261 \mathrm{ff}$. 
Schon durch viele Jahrzehnte hindurch finden sich in kirchlichen Kreisen verschiedenartige, durch angelsächsische Erweckungschristlichkeit beeinflußte Strömungen. Auf diesem Boden waren schon zwei Organisationen aktiv, die Anhänger in verschiedenen Landesteilen, besonders in Städten hatten.

Der ideologische Umbruch der 60er Jahre bildete im allgemeinen eine Bedrohung für die gesamte religiöse Weltschau. Diese Bedrohung vereinigte die bis dahin teilweise recht uneinheitlichen fundamentalistischen Kreise. Unter deren Einwirkung ereignete sich die Organisierung der neupietistischen Bewegung nach der Mitte der 60er Jahre recht schnell. Am Anfang stand die schon erwähnte Geburt der Studentenmission 1964. Eine eigene Zeitung, die relativ schnell eine ziemlich weite Verbreitung erreichte, wurde 1965 gegründet. Eine Dach- und Kooperationsorganisation entstand unter dem Namen Volksmission $1967^{11}$. In den folgenden Jahren wurden Parallelorganisationen für Kinder-, Jugend- und Schülermission wie auch für allgemeine Mission sowie für Slavenmission gegründet.

Besonders Ende der 60er Jahre waren die charismatischen und ethusiastischen Züge der Bewegung sehr deutlich ersichtlich. Kritik an der Kirche wurde ziemlich schroff geübt. Seit Ende der 60er Jahre kam in der Mitte der Anhängerschar stellenweise politische Aktivierung vor. Die damals entstandene Partei, der Finnische Christliche Verband, wurde nämlich von dieser Seite unterstützt, allerdings auch ziemlich stark von sektiererischen Kreisen. In den politischen Tendenzen des Christlichen Verbandes hat man die Forderung nach Wiederherstellung der christlichen Einheitskultur gesehen. Obwohl die Partei für die Anhänger der Volksmission deutlich ein Weg zur Politik gewesen ist, begrenzt sich der Boden der Sympathie nicht nur auf diese Bewegung.

Die Volksmission wurde anfänglich als Kooperations- und Dachorganisation der erweckungschristlichen Kreise gegründet. Ziemlich schnell bildete sich daraus allerdings eine Erweckungsbewegung mit eigenem Gepräge. Die eigenständige Institutionalisierung und Organisierung der Bewegung wurde einerseits von der Kritik gegen die Kirche beschleunigt. Andererseits akzentuierte sich ihre Eigenständigkeit wegen der Kritik der Bewegung gegenüber anderen Erweckungsbewegungen.

Beim Eintritt in die Mitte der 70er Jahre hat sich die gesellschaftliche

11 Cf. Kauppinen $12 \mathrm{ff}$; Sihvo $264 \mathrm{ff}$. 
Situation vom Standpunkt religiöser Aktivität her verändert. Die sichtbare Bedrohung ist schwächer als zuvor. Als Folge davon steht die teilweise Desintegration der Volksmission. Die äußere Bedrohung unterstützt nicht die Zusammenarbeit verschiedener fundamentalistischer Kreise, und so hat sich denn die Bewegung auch schon in zwei Hälften gespalten.

Anders als die traditionellen finnischen Erweckungsbewegungen hat die Volksmission zuerst in den Städten und erst allmählich in gewissem Maße auch auf dem Lande Anklang gefunden ${ }^{12}$. Sie hatte Rückhalt besonders in wachsenden Städten, in denen die traditionelle Kirchlichkeit schwach war. In ländlichen Gegenden war ihr Anklang am stärksten in Gebieten, in denen die alten Erweckungsbewegungen nur sehr wenig wirksam gewesen sind. Als sich die Volksmission organisierte, ist sie moderner gewesen als andere vergleichbare Einrichtungen. Die organisatorischen Grundeinheiten umfassen gewöhnlich eine ganze Landschaft, und kein Dorf oder Stadtteil, manchmal nicht einmal ein Standort solcher gemeindlichen Grundeinheiten hat in der lokalen Organisation besondere Bedeutung.

\section{Traditionelle Erweckungsbewegungen}

Die alten pietistischen Erweckungsbewegungen in Finnland, die pietistische Bewegung, die evangelische Bewegung, die laestadianische Bewegung und die Beterbewegung haben mit dem Wandel der Gesellschaft eine Abschwächung der Voraussetzungen ihrer Aktivität erfahren müssen. Von alters her haben sie ihren Rückhalt zur Hauptsache auf dem Lande gefunden, aber die Landflucht hat den Bevölkerungsschwerpunkt in die Städte verlagert. Allerdings hat sie dabei auch die Anhänger der Bewegung aus ländlichen Gegenden in die Städte gebracht. Teilweise aus diesem Grunde haben besonders die Laestadianer in den letzten Jahren anscheinend eine Ausweitung ihres regionalen Rückhaltes erfahren. Das Einzugsgebiet der evangelischen und der pietistischen Bewegung hat sich trotz der Landflucht nicht erweitert ${ }^{13}$.

Dort, wo Erweckungsbewegungen in Städten aktiv sind, ist diese Aktivität ihrer Natur nach anders beschaffen als auf dem Lande. Die Anhängerschar bildet einen viel isolierteren Kreis. Die gesellschaftlichen, unmittelbaren Einflüsse sind gleichfalls viel schwächer als auf dem Lande.

\footnotetext{
${ }^{12}$ Cf. Kauppinen 3; Sihvo 266.

${ }^{13}$ Cf. Sihvo 261.
} 
Erweckungsbewegungen sind in der soziologischen Forschung meist als Protestbewegungen behandelt worden. Untersucht worden ist deren Kritik an der vorherrschenden Kirchlichkeit und an der Gesellschaft. Weniger Beachtung geschenkt worden ist allerdings deren Charakter als expressives Beieinandersein bietende Kleingruppen und als religiöse Bezugsgruppen. Die Kleingruppe der Erweckungsbewegung stellt für den in die Stadt Ziehenden eine Anpassungshilfe an die neue Umwelt dar. Sie bedeutet jenen Kontinuität des Wertgefüges und Normensystems, die auch in den Städten danach trachten, an solcherlei Aktivität Anteil zu haben.

Aber Erweckungsbewegungen sind durchaus in den 60er Jahren fortwährend als Protestbewegungen aufgetreten. Die Wellen des Radikalismus hoben deren Protest hervor mit teilweise neuen Akzenten. Ihre gesellschaftlichen Stellungnahmen haben zugenommen. In denen herrschte die Tendenz vor, derartige Normen zu unterstützen, welche die Integrität der Familien und nüchterne Lebensweisen festigen.

Der ideelle Umbruch der 60er Jahre brachte es auch zustande, daß unter den Anhängern der Bewegungen Kooperation entstand gegen die Anpassung der Kirche und gegen die allgemeine Ausweitung und damit Abschwächung der Normen. Die sogenannte Bekenntnisbewegung fand Anklang auch seitens der Neupietisten. Als sich die Bedrohung gegen das religiöse Wertgefüge Mitte der 70er Jahre dann abschwächt, ist die Bekenntnisbewegung, die auf seiten verschiedener Bewegungen Anklang gefunden hatte, praktisch gesehen erloschen. Auf die Betonung der Konfessionalität ist jedoch innerhalb der Bewegungen, besonders aber in der evangelischen Bewegung, ständig Gewicht gelegt worden.

\section{Außere Reaktionen}

Meiner Meinung nach ist es überraschend, daß die Sekten ihre Anhängerschaft im Finnland der 60er und 70er Jahre nicht nennenswert erweitert haben. Die Pfingstbewegung und die Zeugen Jehovas sind wohl erfolgreicher gewesen als andere, aber im allgemeinen haben die Sekten keine Reaktion auf die soziale Veränderung dargestellt. Als Ursache dafür steht wohl die oben schon öfters erwähnte Bedrohung, die gegen das ganze religiöse System gespürt wurde. Sie hat verschiedene religiöse Kreise mehr verbunden als aufgelöst.

In den 70er Jahren haben freilich neue, im besonderen aus dem Orient stammende religiöse Bewegungen in den größeren Städten Anklang ge- 
funden ${ }^{14}$. Auf christlicher Seite haben dagegen charismatische Phänomene wellenförmig zugenommen. Es ist allerdings bemerkenswert, daß diese erst in dern 70er Jahren in größeren Zügen aufgetreten sind, als der äußere Druck gegen das religiöse System schon nachließ.

Wie in den Erweckungsbewegungen, so ereignete sich auch im Protest der Sekten Ende der 60er Jahre eine gewisse Politisierung. Deren Anhänger hatten früher fast gar kein Interesse gegenüber Politik und gesellschaftlicher Aktivität gezeigt. Die entstandene neue christliche Partei ist dennoch auch für die Anhänger von Sekten ein Weg zu politischer Betätigung.

Wie bekannt, wird die Institutionalisierung des Protestes der Sekten gewöhnlich Verkirchlichung genannt. Man könnte aber wohl dennoch nicht sagen, daß in Finnland Verkirchlichung von Sekten stattgefunden hat. Sekten haben sich als Sekten institutionalisiert. An deren Verkirchlichung hat die vielleicht wichtigste Voraussetzung gefehlt, nämlich die Ausweitung der Anhängerschaft.

\section{Literaturverzeichnis}

Glock, C., Stark, R., 1965, Religion and Society in Tension. Chicago.

Höllinger, S., 1972, Das Verhältnis der Jugend zur Religion am Aliàng der siebziger Jahre. Religion im Umbruch. Stuttgart.

Kauppinen, J., 1972, An Evangelising Revival Movement. Publications of the Research Institute of the Finnish Lutheran Church 11. Tampere.

Koskelainen, O., 1968, Religiosity in Helsinki. Temenos 3.

Lindqvist, M., 1975, Der lange Weg zur Veränderung. Evangelische Kommentare 8.

Luckmann, T., 1963, Das Problem der Religion in der modernen Gesellschaft. Freiburg.

Pentikäinen, J., Revivalist Movements and Religious Contracultures in Finland. Scripta Instituti Donneriani Aboensis 7. Stockholm.

Sentzke, G., 1963, Die Kirche Finnlands. Helsinki.

Seppänen, P., Finland. Western Religion Country by Country. The Hague.

Sihvo, J., 1973, Expanding Revival Movements and their Circumstances in Finland. Acts of the I2th International Conference on Sociology of Religion. Lille.

Singer, G., 1972, Sozio-Kulturelle Determinanten religiöser Orientierung. Religion im Umbruch. Stuttgart.

Yinger, J., 1971, The Scientific Study of Religion. New York.

${ }^{14}$ Cf. Pentikäinen $114 \mathrm{ff}$. 